\title{
Resource limitation determines realized thermal performance and the potential for metabolic meltdown.
}

\author{
Anna Vinton ${ }^{1}$ and David Vasseur ${ }^{2}$ \\ ${ }^{1}$ Oxford University \\ ${ }^{2}$ Yale University
}

November 22, 2021

\begin{abstract}
1) As temperatures rise across the globe, many species may approach or even surpass their physiological tolerance to withstand high temperatures. Thermal performance curves, which depict how vital rates vary with temperature, are often measured under ideal laboratory conditions and then used to determine the physiological or demographic limits of persistence. However, this approach fails to consider how interactions with other factors (e.g. resources, water availability) may buffer or magnify the effect of temperature change. Recent work has demonstrated that the breadth and shape of a consumer's thermal performance curve change with resource densities, highlighting the potential for temperature interactions and leading to a potential 'metabolic meltdown' when resources decline during warming (Huey and Kingsolver 2019). 2) Here, we further develop the basis for the interaction between temperature and resource density on thermal performance, persistence, and population dynamics by analyzing consumer-resource dynamic models. We find that the coupling of consumer and resource dynamics relaxes the potential for metabolic meltdown because a reduction in top-down control of resources occurs as consumers approach the limits of their thermal niche. However, when both consumers and resources have vital rates that depend on temperature, asymmetry between their responses can generate the necessary conditions for metabolic meltdown. 3) Moreover, we define the concept of a 'realized' thermal performance curve that takes into account the dynamic interaction between consumers, resources and temperature, and we describe an important role for this concept moving forward. 4) Synthesis. A better understanding of the link between temperature change, species interactions, and persistence allows us to improve forecasts of community response to climate change. Our work elucidates the importance of thermal asymmetries between interacting species, and resource limitation as a key ingredient underlying realized thermal niches.
\end{abstract}

\section{Hosted file}

ecollet.docx available at https://authorea.com/users/447395/articles/546394-resourcelimitation-determines-realized-thermal-performance-and-the-potential-for-metabolicmeltdown 